\title{
PRECIPITAÇÃO PLUVIAL MENSAL EM NÍVEIS DE PROBABILIDADE PELA DISTRIBUIÇÃO GAMA PARA DUAS LOCALIDADES DO SUDOESTE DA BAHIA
}

\author{
Monthly rainfall in levels of probability by the gamma distribution for two places \\ of the southwest of Bahia state
}

\author{
Rogério Mendes Murta ${ }^{1}$, Sônia Martins Teodoro ${ }^{2}$, Paulo Bonomo ${ }^{3}$, Modesto Antônio Chaves ${ }^{4}$
}

\begin{abstract}
RESUMO
Com este trabalho objetivou-se testar o modelo de Distribuição Gama na estimativa da precipitação pluvial mensal para Itapetinga e Vitória da Conquista-BA. Utilizaram-se duas bases de dados pluviais mensais do período de 1978 a 1997 para a cidade de Itapetinga e, de 1985 a 1995 para a cidade de Vitória da Conquista. Para cada base de dados determinaram-se os parâmetros a e b da Distribuição Gama para a probabilidade mensal de chuva. As distribuições ajustadas foram usadas para estimar as probabilidades de chuva para cada mês, comparando-as aos valores observados, para o período de 1998 a 2002 em Itapetinga e de 1995 a 2002 em Vitória da Conquista. Para verificar o ajuste dos dados estimados aos observados, utilizou o teste de aderência de KolmogorovSmirnov (2003), cujo resultado foi significativo $(\mathrm{p}<0,01)$. Concluiu-se que a Distribuição Gama apresentou bom ajuste aos dados de Itapetinga e Vitória da Conquista-BA, possibilitando sua utilização para estimativas de precipitação pluvial provável mensal em diferentes níveis de probabilidade.
\end{abstract}

Termos para indexação: Modelos Probabilísticos, Aderência de Distribuições de Probabilidade de Chuva, Estatísticas de Chuva.

\begin{abstract}
This work was made aiming at testing the use of the Gamma Distribution model to estimate monthly rainfalls for the cities of Itapetinga and Vitória da Conquista in the Bahia state in Brazil. Two monthly rainfall data bases were used in the model construction. The first data base held information for the years from 1978 to 1997 for the city of Itapetinga. The second one held information from 1985 to 1995 for the city of Vitória da Conquista. Using each one of these data bases a and b parameters of the Gamma Distribution, for monthly rainfalls, were determinate. The adjusted distributions were used to estimate rainfall probabilities for each month of the year. The estimated values of the average monthly rainfall were compared to observed values for the period from 1998 to 2002 at Itapetinga and from 1995 to 2002 at Vitória da Conquista. The kolmogorov-Smirnov (2003) test was used in order to verify the adjustment of the estimated data to the observed values and the result was significant $(\mathrm{p}<0.01)$. It was concluded that the values estimated, using the Gamma Distribution, showed a good adjustment in relation to the measured values for both cities, Itapetinga and Vitória da Conquista, and that this estimated values can be use in order to generate synthetic data for monthly rainfalls and monthly rainfalls probabilities in these places.
\end{abstract}

Index terms: Probabilistic Models, Probability Rainfalls Distributions Adherence, Rain Statistics.

(Recebido para publicação em 20 de dezembro de 2003 e aprovado em 7 de julho de 2005)

\section{INTRODUÇÃO}

Uma das preocupações quanto às chuvas é a intensidade e a frequiência de suas ocorrências, pelos seus efeitos potencialmente danosos, quando em excesso ou por escassez. O conhecimento das probabilidades de ocorrência de chuva é de suma importância no planejamento agrícola, possibilitando o plantio em época adequada, o planejamento de irrigação, aplicação de adubos e defensivos, a utilização de máquinas agrícolas, etc. É por meio da agrometeorologia operacional que se pode obter valores de estimativas que irão permitir tomadas de decisão, tais como: preparo do solo, semeadura, irrigação e colheita (CHAVES, 1999).

A precipitação pluvial de um determinado local pode ser estimada, dentre outras formas, em termos probabilísticos, mediante modelos teóricos de distribuição ajustados a uma série de dados. Os modelos gerados, após a comprovação da aderência dos dados à distribuição teórica, podem fornecer informações úteis para o planejamento de muitas atividades (FIETZ et al., 1997).

Segundo Cunha et al. (1997), um modelo que vem sendo bastante citado na literatura é o da Distribuição Gama. Seu uso se prende ao fato de que as precipitações,

\footnotetext{
1 Graduando em Zootecnia Universidade Estadual da Sudoeste da Bahia, Bolsista de Iniciação Científica/FAPESB - murta81@bol.com.br

${ }^{2}$ Zootecnista, Professora Doutora do Departamento de Estudos Básicos e Instrumentais - Universidade Estadual da Sudoeste da Bahia.

${ }^{3}$ Engenheiro Agrônomo, Professor Doutor do Departamento de Estudos Básicos e Instrumentais - Universidade Estadual da Sudoeste da Bahia.

${ }^{4}$ Engenheiro Agrícola, Professor, Doutor do Departamento de Estudos Básicos e Instrumentais - Universidade Estadual da Sudoeste da Bahia.
} 
sob o ponto de vista estatístico, não são distribuídas uniformemente em torno do valor médio, mas sim, de maneira irregular, com grande desvio em relação a este valor médio (KREPPER et al., 1989). Vários estudos mostram que a Distribuição Gama representa adequadamente o comportamento da chuva, dentre os quais podem ser citados aqueles realizados por Castro (1996), Cunha et al. (1997) e Thom (1958). Castro (1996) verificou que a Distribuição Gama foi adequada para estimar a probabilidade de precipitação em Viçosa-MG e enfatizou que os projetos de irrigação não devem ser baseados nos valores médios de precipitação porque estes, em geral, apresentaram menos de $50 \%$ de probabilidade de ocorrência.

Thom (1958) concluiu que a Distribuição Gama pode ser considerada como a mais adequada para períodos curtos de chuva.

Estudos de ajustes de função de distribuição de probabilidade ou estimativas de probabilidade usando funções de distribuição de probabilidade teóricas em relação a um conjunto de elementos climáticos têm sido desenvolvidos, enfatizando os benefícios no planejamento de atividades que minimizem riscos climáticos. Entre estes podemos citar: precipitação pluvial (ASSAD \& CASTRO, 1991; ASSIS, 1991; ASSIS et al., 1996; ÁVILA et al., 1996; BERLATO, 1987; CASTRO, 1996; BOTELHO \& MORAIS, 1999; CATALUNHA et al., 2002), temperatura do ar (BURIOL et al., 2000b; MOTA et al., 1999) e radiação solar (BURIOL et al., 2000a).

Para comparar as probabilidades empíricas de uma variável com as probabilidades teóricas estimadas por uma dada função de distribuição, usam-se testes de aderência, como o $\chi^{2}$, Kolmogorov-Smirnov (2003), Lilliefors, Shapiro-Wilk, Cramervon Mises (ASSIS et al., 1996; CAMPOS, 1983), de forma a verificar se os valores da amostra podem ser considerados como provenientes de uma população com aquela distribuição teórica. Nos testes de aderência, a hipótese nula $\left(\mathrm{H}_{0}\right)$ admite que a distribuição seja a especificada, com os seus parâmetros estimados com base nos dados amostrais (ASSIS et al., 1996; CATALUNHA et al., 2002).

Existem diferenças entre os testes de aderência, quanto à probabilidade de rejeitar $\mathrm{H}_{0}$, quando efetivamente $\mathrm{H}_{0}$ é falsa (1- $\beta$ ). Os testes de $\chi^{2} \mathrm{e}$ de KolmogorovSmirnov (2003) são amplamente utilizados (ASSIS et al., 1996). Já o teste de Lilliefors (CAMPOS, 1983) é mais eficaz que o teste de Kolmogorov-Smirnov (2003), porém é específico para verificar a aderência dos dados amostrais à distribuição normal. Os testes de Shapiro-Wilk e Cramer- von Mises também se diferenciam quanto à sua eficácia (CARGNELUTTI FILHO et al., 2004).

$O$ teste de aderência de $\chi^{2}$ apresenta limitações como o fato da frequiência de uma classe não poder ser inferior a cinco e os dados serem agrupados em classes perdendo informações, o que não ocorre no teste de Kolmogorov-Smirnov (2003). Este último pode ser realizado com os dados agrupados ou, ainda, estes isoladamente, sendo normalmente mais eficiente que o $\chi^{2}$ em pequenas amostras, ou seja, menos de 30 observações (CAMPOS, 1983).

O teste de Kolmogorov-Smirnov (2003) é baseado no módulo da maior diferença entre a probabilidade observada e a estimada, que é comparada com um valor tabelado de acordo com o número de observações da série sob teste (CATALUNHA et al., 2002).

Conforme o exposto, com este trabalho objetivouse testar o modelo de Distribuição Gama para estimativa da precipitação pluvial em Itapetinga e Vitória da Conquista-BA, criando assim, a possibilidade de estimar a precipitação pluvial provável mensal em diferentes níveis de probabilidade.

\section{MATERIAIS E MÉTODOS}

Os dados foram cedidos pela CEPLAC/EMARC-IT (Comissão Executiva do Plano da Lavoura Cacaueira) e pela UESB Campus de Vitória da Conquista, sendo oriundos das respectivas estações meteorológicas e climatológicas.

A estação de Itapetinga está localizada a $15^{\circ} 09^{\prime} 07^{\prime \prime}$ de latitude Sul, $40^{\circ} 15^{\prime} 32^{\prime \prime}$ de longitude Oeste e altitude média de 268 metros, a de Vitória da Conquista está a $14^{\circ} 53^{\prime}$ de latitude Sul, $40^{\circ} 48^{\prime}$ de longitude Oeste e 874 metros de altitude.

Em virtude da disponibilidade de dados, a base de dados de Itapetinga compreendeu o período de 1978 a 1997 e a de Vitória da Conquista o período de 1985 a 1995.

A distribuição acumulada Gama G (y) (THOM, 1958) é dada por:

Os parâmetros $\alpha$ e $\beta$ que propiciam a distribuição Gama acumulada para uma dada variável aleatória foram, estimados pelo método da máxima verossimilhança conforme (ASSIS et al., 1996):

$$
G(y)=\frac{1}{\beta^{\gamma} \times \Gamma(\alpha)} \times \int_{0}^{\gamma} e^{-\gamma / \alpha} \times y^{\gamma-1} d y
$$




$$
\begin{aligned}
& \alpha=\frac{1}{4 A}\left(1+\sqrt{1+\frac{4 A}{3}}\right) \\
& \beta=\frac{\bar{X}}{\alpha} \\
& a=\ln \bar{X}-X_{g}
\end{aligned}
$$

Sendo $\overline{\mathrm{X}}$ e Xg respectivamente, a média aritmética e a média geométrica das observações.

Para cada uma destas bases ajustaram-se os parâmetros $\alpha$ e $\beta$ da Distribuição Gama aos valores mensais de precipitação pluvial. Para valores de a inferiores a 5,6 aplicou-se o fator de correção descrito por Thom (1958). As probabilidades de chuva foram estimadas para cada mês e comparadas com valores reais, para o período de 1998 a 2002, e 1995 a 2002 para Itapetinga e Vitória da Conquista, respectivamente.

Para verificar o ajuste dos dados estimados aos observados, utilizou-se o teste de aderência de KolmogorovSmirnov (2003) ao nível de 1\% de significância. O teste foi realizado via Web na página http://Www.physics.csbsju.eduj istats/K $\bar{S}$-test.html. Na busca de valores atípicos realizou-se o teste de Tukey a $1 \%$ de significância.

\section{RESULTADOS E DISCUSSÕES}

Das bases de dados estudadas obteve-se para a cidade de Itapetinga-BA a precipitação pluvial anual média de 867 mm, sendo o ano mais chuvoso o de 1992, com uma precipitação pluvial de $1342 \mathrm{~mm}$ e o menos chuvoso, o de 1996, com uma precipitação pluvial de $448 \mathrm{~mm}$. Para a cidade de Vitória da Conquista a precipitação anual média foi de $741 \mathrm{~mm}$, sendo o ano mais chuvoso o de 1985, com uma precipitação de 1188 mm e o menos chuvoso o de 1990, com $367 \mathrm{~mm}$ de precipitação. Estes valores estão dentro das faixas previstas, para a região, nas normas climatológicas publicadas pelo INMET (2005). Observouse que, ao longo de todo o ano a precipitação em Itapetinga foi maior que a de Vitória da Conquista, com exceção dos meses de março e dezembro, o que está de acordo com os valores obtidos por Murta et al. (2004).

As estimativas dos parâmetros $\alpha$ e $\beta$ da Distribuição Gama ajustada, são apresentados na Tabela 1 para as duas cidades.

Podemos observar que os valores do parâmetro $b$ não excederam o valor 100 em nenhum mês, possibilitando assim a utilização na Distribuição Gama para o cálculo da estimativa das precipitações prováveis para Itapetinga e Vitória da Conquista-BA. De acordo com Thom (1958), para valores superiores a 100 não se utiliza a Distribuição Gama incompleta.

TABELA 1 - Valores de $\alpha$ e $\beta$ para Itapetinga e Vitória da Conquista-BA.

\begin{tabular}{lrrrr}
\hline \multirow{2}{*}{ MÊS } & \multicolumn{2}{c}{ ITAPETINGA } & \multicolumn{2}{c}{ VITÓRIA DA CONQUISTA } \\
\cline { 2 - 5 } & $\boldsymbol{\alpha}$ & $\boldsymbol{\beta}$ & $\boldsymbol{\alpha}$ & $\boldsymbol{\beta}$ \\
\hline Janeiro & 1,80 & 57,14 & 1,56 & 67,33 \\
Fevereiro & 1,84 & 38,68 & 1,52 & 37,01 \\
Março & 1,97 & 63,95 & 1,60 & 56,70 \\
Abril & 1,94 & 46,99 & 2,04 & 21,02 \\
Maio & 1,99 & 21,42 & 1,94 & 11,36 \\
Junho & 2,59 & 19,29 & 2,17 & 12,12 \\
Julho & 2,36 & 15,01 & 1,77 & 12,85 \\
Agosto & 14,19 & 1,70 & 14,01 \\
Setembro & 1,09 & 27,03 & 1,77 & 12,57 \\
Outubro & 1,59 & 27,45 & 1,64 & 33,30 \\
Novembro & 2,15 & 50,12 & 1,78 & 68,79 \\
Dezembro & 2,14 & 59,58 & 1,96 & 88,42 \\
\hline
\end{tabular}

Ciênc. agrotec., Lavras, v. 29, n. 5, p. 988-994, set./out., 2005 
Os maiores valores de $\alpha$ foram encontrados no período seco e os menores, no período chuvoso. Já para o parâmetro $\beta$ os maiores valores foram encontrados para os períodos de chuvas mais intensas. Estes resultados estão de acordo com Botelho \& Morais (1999), segundo estes autores, isso pode ser explicado pela pronunciada assimetria nos períodos dos meses mais secos, visto que a assimetria é inversamente proporcional a $\alpha$. Por outro lado, Galate (1987), encontrou valores de $\beta$ menores nos meses menos chuvosos. Estimativas da variância aumentaram rapidamente com o crescimento dos valores de $\beta$, sendo este um indicador da variabilidade dos dados, visto que variância é diretamente proporcional ao quadrado de $\beta$ Portanto, os valores maiores de $\beta$ no período chuvoso, aqui encontrados, indicam uma maior variabilidade dos dados, nos meses mais chuvosos.

A Tabela 2 mostra as probabilidades de precipitação pluvial mensal provável para Itapetinga, nos níveis de 10, 20, 30, 40, 50, 60, 70, 80, 90\% estimados pela Distribuição Gama.

Estes níveis referem-se à probabilidade específica de ocorrência de uma precipitação mínima provável. Por exemplo, em Itapetinga-BA, para o mês de janeiro, existe a probabilidade de $10 \%$ da precipitação acumulada ser igual ou superior a $205 \mathrm{~mm}$, ou seja, num intervalo de 10 anos que em um ano ocorra pelo menos $205 \mathrm{~mm}$ no mês de janeiro.

Pela Tabela 3 observa-se as probabilidades de precipitação pluvial mensal provável para Vitória da Conquista, nos níveis de 10, 20, 30, 40, 50, 60, 70, 80, 90\% estimados pela Distribuição Gama.

Na Tabela 4 exibe-se a probabilidade de ocorrência da precipitação pluvial média para Itapetinga e Vitória da Conquista-BA.

Segundo Castro (1996), no Brasil é comum se adotar valores médios de precipitação pluvial como parâmetros de dimensionamento de projetos agrícolas. Segundo Marquelli et al. (1988), citados por Castro (1996), a ocorrência do valor médio de precipitação pluvial mensal se dá entre os níveis de 40 e $50 \%$ de probabilidade, esses níveis são inferiores em relação ao nível de $75 \%$ de probabilidade, geralmente recomendado na elaboração de projetos agrícolas. Os valores encontrados neste trabalho, para a probabilidade de ocorrência de valores médios de precipitação pluvial mensal, ocorreram próximos ao nível de $40 \%$ de probabilidade, confirmando o que afirmam estes autores.

Aplicando o teste de Kolmogorov-Smirnov (2003) para avaliar o ajuste dos dados de precipitação média, estimados pela Distribuição Gama, aos valores observados nos anos de 1998 a 2002 para Itapetinga e 1995 a 2002 para Vitória da Conquista, confirmou-se o ajuste, para ambas, a $1 \%$ de probabilidade.

Pelo teste de Tukey, na busca de valores atípicos, encontrou-se que os valores de precipitação média de $313,279,238,213,211,193$, para Itapetinga se enquadram nesta categoria, correspondendo a novembro de 1999 , janeiro de 2002, novembro de 1998, abril de 2000, dezembro de 1999 e fevereiro de 2002, respectivamente.

TABELA 2 - Probabilidades mensais de ocorrência de chuvas para Itapetinga-BA, estimadas pela Distribuição Gama.

\begin{tabular}{|c|c|c|c|c|c|c|c|c|c|}
\hline \multirow{2}{*}{ MÊS } & \multicolumn{9}{|c|}{ Probabilidade de Chuva (mm) Itapetinga-BA } \\
\hline & $10 \%$ & $20 \%$ & $30 \%$ & $40 \%$ & $\mathbf{5 0 \%}$ & $60 \%$ & $70 \%$ & $80 \%$ & $90 \%$ \\
\hline Janeiro & 205,0 & 156,0 & 125,5 & 103,0 & 84,5 & 68,5 & 54,0 & 39,5 & 24,5 \\
\hline Fevereiro & 141,0 & 107,5 & 87,0 & 71,5 & 59,0 & 47,5 & 37,5 & 28,0 & 17,5 \\
\hline Março & 246,0 & 189,0 & 153,5 & 127,0 & 105,5 & 86,5 & 68,5 & 51,5 & 33,0 \\
\hline Abril & 157,0 & 118,0 & 94,0 & 76,5 & 62,0 & 49,5 & 38,5 & 27,5 & 16,5 \\
\hline Maio & 83,0 & 64,0 & 52,0 & 43,0 & 36,0 & 29,5 & 23,5 & 17,5 & 11,5 \\
\hline Junho & 91,5 & 72,5 & 60,5 & 51,5 & 44,0 & 37,0 & 30,5 & 24,0 & 16,5 \\
\hline Julho & 66,5 & 52,0 & 43,0 & 36,0 & 30,5 & 25,5 & 21,0 & 16,0 & 11,0 \\
\hline Agosto & 57,0 & 44,0 & 36,0 & 30,0 & 25,0 & 21,0 & 16,5 & 12,5 & 8,0 \\
\hline Setembro & 88,5 & 66,0 & 52,5 & 42,5 & 34,5 & 27,5 & 21,0 & 15,0 & 9,0 \\
\hline Outubro & 113,0 & 87,5 & 72,0 & 60,0 & 50,0 & 41,5 & 33,5 & 25,5 & 17,0 \\
\hline Novembro & 205,5 & 159,5 & 131,0 & 109,0 & 91,5 & 75,5 & 61,0 & 46,0 & 30,5 \\
\hline Dezembro & 226,5 & 174,8 & 141,0 & 116,5 & 96,5 & 79,0 & 62,5 & 47,0 & 30,0 \\
\hline
\end{tabular}


MURTA, R. M. et al.

TABELA 3-Probabilidades mensais de ocorrência de chuvas para Vitória da Conquista-BA, estimados pela Distribuição Gama.

\begin{tabular}{|c|c|c|c|c|c|c|c|c|c|}
\hline \multirow{2}{*}{ MÊS } & \multicolumn{9}{|c|}{ Probabilidade de chuva (mm) Vitória da Conquista-BA } \\
\hline & $10 \%$ & $20 \%$ & $30 \%$ & $40 \%$ & $50 \%$ & $60 \%$ & $70 \%$ & $80 \%$ & $90 \%$ \\
\hline Janeiro & 217,0 & 162,0 & 128,5 & 103,5 & 84,0 & 66,5 & 51,0 & 36,5 & 21,5 \\
\hline Fevereiro & 117,0 & 87,0 & 69,0 & 55,5 & 44,5 & 35,0 & 27,0 & 19,0 & 11,0 \\
\hline Março & 186,0 & 139,5 & 111,0 & 90,0 & 72,5 & 58,0 & 44,5 & 32,0 & 19,0 \\
\hline Abril & 83,0 & 64,0 & 52,0 & 43,5 & 36,0 & 29,5 & 23,5 & 18,0 & 11,5 \\
\hline Maio & 43,5 & 33,0 & 27,0 & 22,5 & 18,5 & 15,0 & 12,0 & 9,0 & 5,5 \\
\hline Junho & 50,5 & 39,0 & 32,0 & 27,0 & 22,5 & 18,5 & 15,0 & 11,5 & 7,5 \\
\hline Julho & 45,5 & 34,5 & 28,0 & 23,0 & 18,5 & 15,0 & 12,0 & 9,0 & 5,5 \\
\hline Agosto & 48,0 & 36,5 & 29,0 & 23,5 & 19,5 & 15,5 & 12,0 & 9,0 & 5,5 \\
\hline Setembro & 44,5 & 34,0 & 27,0 & 22,0 & 18,0 & 14,5 & 11,5 & 8,5 & 5,5 \\
\hline Outubro & 111,5 & 84,0 & 67,0 & 54,5 & 44,0 & 35,5 & 27,5 & 19,5 & 12,0 \\
\hline Novembro & 245,5 & 186,0 & 150,0 & 123,0 & 100,5 & 81,5 & 64,0 & 47,0 & 29,0 \\
\hline Dezembro & 338,5 & 260,0 & 211,5 & 175,0 & 145,0 & 118,5 & 94,0 & 70,5 & 45,5 \\
\hline
\end{tabular}

TABELA 4 - Probabilidade de ocorrência da precipitação pluvial média para Itapetinga e Vitória da Conquista-BA.

\begin{tabular}{lcccc}
\hline \multirow{2}{*}{ MÊS } & \multicolumn{3}{c}{ Probabilidade de Ocorrência da Precipitação Pluvial Média } \\
\cline { 2 - 5 } & \multicolumn{2}{c}{ Itapetinga } & \multicolumn{2}{c}{ Vitória da Conquista } \\
\cline { 2 - 5 } & Média & Probabilidade (\%) & média & Probabilidade (\%) \\
\hline Janeiro & 103,0 & 40,1 & 105,1 & 39,4 \\
Fevereiro & 71,2 & 40,2 & 56,2 & 39,2 \\
Março & 125,9 & 40,5 & 90,7 & 39,5 \\
Abril & 77,1 & 39,6 & 42,8 & 40,7 \\
Maio & 42,7 & 40,6 & 22,1 & 40,5 \\
Junho & 50,0 & 41,7 & 26,4 & 41,0 \\
Julho & 35,5 & 41,3 & 22,8 & 40,0 \\
Agosto & 29,7 & 40,8 & 23,8 & 39,8 \\
Setembro & 43,1 & 39,5 & 22,2 & 40,0 \\
Outubro & 59,1 & 40,9 & 54,7 & 39,6 \\
Novembro & 107,5 & 40,9 & 122,7 & 40,1 \\
Dezembro & 115,7 & 40,5 & 173,3 & 40,5 \\
\hline
\end{tabular}

Portanto, estes períodos podem ser considerados como de chuvas atípicas, em relação ao período total de análise, mostrando a normalidade da ocorrência de chuvas mais intensas no verão. Para Vitória da Conquista encontraramse como valores atípicos de precipitação as médias 376 , $351,306,239$, correspondendo a dezembro de 2000, março de 1997, janeiro de 2002 e dezembro de 1995, respectivamente, confirmando a normalidade no verão.

\section{CONCLUSÃO}

Os resultados encontrados permitem concluir que a Distribuição Gama apresentou bom ajuste aos dados de chuva para as duas localidades estudadas, no sudoeste da Bahia, podendo, portanto, ser usada para estimativas da precipitação pluvial provável mensal em diferentes níveis de probabilidade. 


\section{REFERÊNCIAS BIBLIOGRÁFICAS}

ASSAD, E. D.; CASTRO, L. H. R. Análise freqüêncial da pluviometria para a estação de Sete Lagoas, MG. Pesquisa Agropecuária Brasileira, Brasília, v. 26, p. 397-402, 1991.

ASSIS, F. N. Modelagem de ocorrência e da quantidade de chuva e dias secos em Piracicaba - SP e Pelotas-RS. 1991. $134 \mathrm{f}$. Tese (Doutorado em Agronomia) - Escola Superior de Agricultura de Luiz de Queiroz, Piracicaba, 1991.

ASSIS, F. N.; ARRUDA, H. V. de; PEREIRA, A. R. Aplicações de estatística à climatologia: teoria e prática. Pelotas: UFPel, 1996. 161 p.

ÁVILA, A. M. H.; BERLATO, M. A.; SILVA, J. B.; FONTANA, D. C. Probabilidade de ocorrência de precipitação pluvial mensal igual ou maior que a evapotranspiração potencial para a estação de crescimento das culturas de primavera-verão no Estado do Rio Grande do Sul. Pesquisa Agropecuária Gaúcha, Porto Alegre, v. 2, p. 149-154, 1996.

BERLATO, M. A. Modelo de relação entre o rendimento de grãos da soja e o déficit hídrico para o Estado do Rio Grande do Sul. 1987. 93 f. Tese (Doutorado) - Instituto Nacional de Pesquisas Espaciais, São José dos Campos, 1987.

BOTELHO, V. A.; MORAIS, A. R. Estimativas dos parâmetros da distribuição gama de dados pluviométricos do Município de Lavras, Estado de Minas Gerais. Ciência e Agrotecnologia, Lavras, v. 23, p. 697-706, 1999.

BURIOL, G. A.; ESTEFANEL, V.; ANDRIOLO, J. L.; MATZENAUER, R.; TAZZO, I. F. Disponibilidade de radiação solar para o cultivo do tomateiro durante o inverno no Estado do Rio Grande do Sul. Pesquisa Agropecuária Gaúcha, Porto Alegre, v. 6, p. 113-120, 2000a.

BURIOL, G. A.; HELDWEIN, A. B.; ESTEFANEL, V.; MATZENAUER, R.; MARCON, I. A. Condições térmicas para o cultivo do pepineiro na região do baixo vale do Taquari, RS: 1. temperaturas baixas limitantes. Pesquisa Agropecuária Gaúcha, Porto Alegre, v. 6, p. 205-213, 2000b.

CAMPOS, H. de. Estatística experimental nãoparamétrica. 4. ed. Piracicaba: ESALQ, 1983.349 p.
CASTRO, R. Distribuição probabilística de precipitação na região de Botucatu-SP. 1996. 88 f. Dissertação (Mestrado em Agronomia) - Universidade Estadual Paulista, Botucatu, 1996.

CARGNELUTTI FILHO, A.; MATZENAUER, R.; TRINDADE, J. K. Ajustes de funções de distribuição de probabilidade à radiação solar global no Estado do Rio Grande do Sul. Pesquisa Agropecuária Brasileira, Brasília, v. 39, n. 12, p. 1157-1166, dez. 2004.

CATALUNHA, M. J.; SEDIYAMA, G. C.; LEAL, B. G.; SOARES, C. P.; RIBEIRO, A. B. Aplicação de cinco funções densidade de probabilidade a séries de precipitação pluvial no Estado de Minas Gerais. Revista Brasileira de Agrometeorologia, Santa Maria, v. 10, n. 1, p. 153-162, 2002.

CHAVES, M. A. Sistema de informação e modelagem de radiação solar. 1999. 237 f. Tese (Doutorado em Agronomia/ Energia na Agricultura) - Universidade Estadual Paulista, Botucatu, 1999.

CUNHA, A. R.; MARTINS, D.; PASSOS, J. R. S. O modelo gama de probabilidade aplicado ao estudo da distribuição da chuva mensal na região administrativa de Bauru-SP. In: CONGRESSO BRASILEIRO DE AGROMETEOROLOGIA, 1997, Campos do Jordão. Anais... Campos do Jordão: SBA, 1997. p. 107-110.

FIETZ, C. R.; URCHEI, M. A.; FRIZZONE, J. A.; FOLEGATTI, M. V. Probabilidade de ocorrência de períodos secos e chuvosos na região de Dourados, MS. In: CONGRESSO BRASILEIRO DE AGROMETEOROLOGIA, 1997, Piracicaba. Anais... Piracicaba: SBA, 1997. v. 1, p. 101-103.

GALATE, R. S. Estudo das precipitações pluviais no município de Belém - PA, através da distribuição gama. 1987. 70 f. Dissertação (Mestrado em Estatística e Experimentação Agronômica) - Escola Superior de Agricultura de Luiz de Queiroz, Piracicaba, 1987.

INMET. Normais climatológicas: 1931-1990. Disponívelem: $x^{\prime} h t=0$

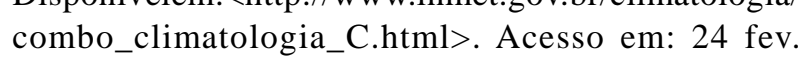
2005 . 
KOLMOGOROV-SMIRNOV. Disponível em: <http:// WWw.physics.csbsju.edu/stats/KS-test.html 30 nov. 2003.

KREPPER, C. M.; SCIAN, B. V.; PIERINI, J. O. Time and space variability of rainfall in central East Argentina. Journal of Climate, [S.1.], v. 2, p. 39-47, 1989.

MOTA, F. S.; ROSSKOFF, J. L. C.; SILVA, J. B. Probabilidade de ocorrência de dias com temperaturas iguais ou superiores a $35^{\circ} \mathrm{C}$ no florescimento do arroz no Rio Grande do Sul. Revista Brasileira de Agrometeorologia, Santa Maria, v. 7, p. 147-149, 1999.

MURTA, R. M.; TEODORO, S. M.; GUSMÃO FILHO, J. D.; CHAVES, M. A. Análise da precipitação pluviométrica em Itapetinga e Vitória da Conquista-BA: estatísticas descritivas e comparações. In: CONGRESSO DE PESQUISA E EXTENSÃO DA UESB, 8., 2004, Itapetinga. Anais... Itapetinga: UESB, 2004. CD-ROM.

THOM, H. C. S. A note on the gama distribuition. Monthly Weather Review, Washington, v. 86, p. 117-22, 1958. 